\title{
The Effect of the Enhanced Endpoint of Pulmonary Vein Isolation on the Long-Term Success Rate of Radiofrequency Ablation for Atrial Fibrillation
}

This article was published in the following Dove Press journal: International Journal of General Medicine

\author{
Jianhua Chen ${ }^{\mathrm{I}, *}$ \\ Quanhe Chen ${ }^{2, *}$ \\ Feilong Zhang' \\ Xuehai Chen' \\ Zhe Xu' \\ Qiong Jiang' \\ Xudong Sun' \\ Jinguo $\mathrm{Li}^{\prime}$ \\ Lianglong Chen' \\ Weiwei Wang' \\ 'Department of Cardiology, Fujian \\ Medical University Union Hospital \& \\ Fujian Provincial Institute of Coronary \\ Disease, Fuzhou, People's Republic of \\ China; ${ }^{2}$ Union Clinic Medical College, \\ Fujian Medical University, Fuzhou, \\ People's Republic of China \\ *These authors contributed equally to \\ this work
}

Objective: This study aimed to investigate whether the enhanced endpoint of pulmonary vein isolation (PVI; intravenous injection of adenosine-triphosphate $[\mathrm{ATP}]+$ pacing capture + supplemental ablation) after initial PVI can reduce the long-term recurrence rate of atrial fibrillation (AF) after PVI.

Methods: Patients with paroxysmal or persistent AF undergoing catheter ablation treatment were enrolled in this study and divided into three groups according to the surgical endpoint: (1) group $1(\mathrm{n}=92)$, in which patients were observed for 30 minutes after the initial PVI and pulmonary vein-left atrium (PV-LA) electrical conduction had not recovered; (2) group 2 (n =99), in which patients were observed for 30 minutes after the initial PVI, then intravenously injected with ATP, and PV-LA electrical conduction had not recovered; and (3) group $3(\mathrm{n}=102)$, in which patients were observed for 30 minutes after the initial PVI, then intravenously injected with ATP + treated with ablation line pacing, and the atrium could not be captured.

Results: Patients were followed up for 12 months after the operation. Twenty-eight patients in group 1 (30.4\%), 19 patients in group $2(19.2 \%)$, and 10 patients in group $3(9.8 \%)$ developed a recurrence of AF. The difference between groups 1 and 3 was statistically significant $(\mathrm{p}<0.001)$. At 12 months after the operation, the thickness of the left atrium, the posterior wall of the left ventricle, and the ventricular septum of the three groups of patients were significantly thinner than before the operation. Furthermore, the left ventricular ejection fraction had increased $(\mathrm{p}<0.05$ for all), and the pulmonary artery pressure had decreased ( $\mathrm{p}$ $<0.001)$.

Conclusion: For patients with paroxysmal AF or persistent AF, the enhanced endpoint of PVI after the initial PVI can reduce the long-term recurrence rate of AF after PVI.

Keywords: ablation, pacing, adenosine, atrial fibrillation

\section{Introduction}

Radiofrequency ablation (RFA) is the first-line treatment for atrial fibrillation (AF), and pulmonary vein isolation (PVI) is the basic procedure of RFA for AF. Pulmonary vein-left atrium (PV-LA) electrical conduction recovery is the main cause of recurrence after AF ablation. ${ }^{1}$ The energy of RFA raises the cell's resting potential to a level above the excitatory threshold, rendering the cell incapable of depolarization and conduction. Adenosine can enhance outward potassium currents, promote hyperpolarization of the cell membrane potential, and preserve excitability. It can also activate sodium channels and increase the maximum depolarization rate
Correspondence: Weiwei Wang Department of Cardiology, Fujian Medica University Union Hospital \& Fujian Provincial Institute of Coronary Disease, No. 29 of Xinquan Road, Gulou District, Fuzhou, 35000I, People's Republic of China

Tel +8659186218022

Fax +8659183322156

Email wang_wwedh@163.com
International Journal of General Medicine 2021:14 697-707

submit your manuscript DovePress $\boldsymbol{f}$ in $\boldsymbol{\nabla}$ http://doi.org/10.2147/1/GM.S294033 
in Phase 0 of the cellular action potential. Previous studies have shown that surgical procedures, such as intraoperative intravenous injection of adenosine triphosphate (ATP) or pacing on the ablation line to locate potential electrical conduction followed by supplementary ablation until PVI is repeated, can help reduce long-term AF recurrence. ${ }^{1,2}$ However, there is no direct evidence demonstrating whether the enhanced endpoint of PVI operation - ATP induction + electrical stimulation induction + supplemental ablation - can reduce the long-term recovery of PV-LA electrical conduction and increase the success rate of RFA for AF. In this study, the enhanced endpoint of the PVI procedure of ATP induction + electrical stimulation induction + supplemental ablation was adopted to determine whether this procedure can reduce the long-term recurrence rate of $\mathrm{AF}$ after PVI and to evaluate both the safety of the operation and postoperative structural and functional changes to the left atrium. The aim was to provide a reliable basis for clinical improvement of PVI surgical methods and the long-term success rate of RFA for AF.

\section{Methods}

\section{Subjects}

A total of 366 patients who underwent RFA between January 1, 2017 and February 28, 2019 were enrolled in this study; the study deadline was February 29, 2020. Among them, 307 patients met the inclusion and exclusion criteria. Inclusion criteria for patients were as follows: (1) patients aged $\geq 18$ and $\leq 85$ years; (2) patients diagnosed with paroxysmal AF or persistent AF (AF duration $\leq 12$ months), treated with at least one antiarrhythmic drug that was ineffective, and due to undergo RFA; and (3) the preoperative New York Heart Association (NYHA) cardiac function grade was I or II. Exclusion criteria: (1) patients aged $<18$ or $>85$ years; (2) patients with a preoperative NYHA cardiac function grade of III or IV; (3) patients diagnosed with persistent AF, but the duration of AF was >12 months; (4) patients with a comorbidity of valvular heart disease, hypertrophic cardiomyopathy, primary dilated cardiomyopathy, replacement bioprosthetic or mechanical mitral or aortic valves; (5) patients failed to undergo RFA due to surgical contraindications; (6) patients failed to achieve the planned surgical goal due to surgical complications; (7) patients whose AF could not be terminated after achieving the predetermined surgical goal; and (8) patients could not be followed up within the prescribed time after surgery.
The present study was approved by the ethics committee of the Affiliated Hospital of Fujian Medical University (Approval number 2020KY060). This study was conducted in accordance with the Declaration of Helsinki. All the patients gave informed consent, signed an informed consent form before surgery, and agreed to accept the study protocol for follow-up examinations.

\section{Radiofrequency Ablation (RFA) Procedure}

Local anesthesia was given. A Quadrupole electrode catheter was used to pace in the right ventricle when transient asystole occurred after the adenosine injection. A ten-pole circular electrode catheter (Triguy, APT Medical, Shenzhen, China) was used to record pulmonary vein potentials, a $3.5-\mathrm{mm}$ saline perfusion ablation catheter (THERMOCOOL SMARTTOUCH, Biosense Webster) was used to construct the three-dimensional structure of the left atrium, and ablation was performed. The ablation power was $30-35 \mathrm{~W}$, and the temperature was $43^{\circ} \mathrm{C}$. The rate of saline perfusion during ablation was $17-25 \mathrm{~mL} / \mathrm{min}$.

\section{Research Grouping}

Patients with sinus rhythm after the initial PVI were included; patients with AF after the initial PVI were given $150 \mathrm{~J}$ DC for synchronous electrical cardioversion. Those who could maintain sinus rhythm after electrical cardioversion were included; patients who could not maintain sinus rhythm after electrical cardioversion and required linear ablation of the top of the left atrium or mitral and tricuspid annulus isthmus, coronary sinus ablation, or other ablation procedures were not included. The patients included were randomly divided into three groups, as described below.

\section{Group I (the Control Group)}

Patients whose PV-LA electrical conduction had not recovered after the operation were observed for 30 minutes after the initial PVI. For those whose PV-LA electrical conduction was restored, ablation was performed again continuously until PVI was achieved, and the operation was ended.

\section{Group 2 (the ATP Group)}

Patients whose PV-LA electrical conduction had not recovered after the operation were observed for 30 minutes after the initial PVI then intravenously injected with 30-40 mg of ATP. For those whose PV-LA electrical 
conduction was restored, ablation was performed again until PVI, and then the operation was ended.

\section{Group 3 (the ATP + Electric Stimulation Group)}

Patients were observed for 30 minutes after the initial PVI, then intravenously injected with $30-40 \mathrm{mg}$ of ATP if the electrical conduction of PV-LA had not recovered and pacing on the ablation line (voltage: $5 \mathrm{~V}$; pulse width: 2 $\mathrm{ms}$ ) could not capture the atrium (confirmation of PVI), and then the operation was ended. Re-ablation was performed for patients in whom pacing could capture the atrium until the pacing could not capture the atrium (PVI). After intravenous injection of ATP, if the electrical conduction of PV-LA was restored, ablation was performed again until PVI. Then, if pacing on the ablation line could not capture the atrium, the operation was ended; if pacing could capture the atrium, re-ablation was performed until the pacing could not capture the atrium, and then the operation was ended (see Figure 1 for details).

\section{Postoperative Follow-Up}

All the patients were followed up regularly in the first week after surgery and every 2-4 weeks thereafter. Electrocardiograms (ECGs) were routinely checked. During the entire follow-up period, if the patient had any symptoms of discomfort, they could contact the doctor for follow-up by phone, message, or WeChat, and check either the ECG, the 24-hour ECG Holter or both in real time. The success criterion of the operation was the absence of $\mathrm{AF}$ with a duration $>1$ minute, atrial flutter, or atrial tachycardia during the follow-up period of 3-12 months after surgery. All the patients underwent transthoracic echocardiography before surgery and at 12 months after surgery.

\section{Postoperative Drug Treatment}

Routine anticoagulation treatment was given for three months after the operation. After three months, it was decided whether to continue anticoagulation therapy according to the patient's CHA2DS2VASc score, the follow-up physician's advice, and the patient's willingness to accept treatment. Routine use of antiarrhythmic drugs (propafenone or amiodarone) was prescribed for three months after the operation; after three months, depending on whether the patient had rapid atrial arrhythmia, the antiarrhythmic drugs could be stopped or continued. If necessary, follow-up physicians could choose angiotensinconverting enzyme inhibitors, angiotensin receptor blockers, $\beta$-receptor blockers, or statin therapy according to the patient's condition.

\section{Statistical Analysis}

All the data analyses were based on the principle of processing intention analysis. Count data were expressed as percentages (\%) and compared between groups using Pearson's chi square test or Fisher's exact test. Measurement data were evaluated using a non-parametric test (the Kolmogorov-Smirnov test). Normally distributed measurement data were expressed as mean \pm standard deviation $(x \pm \mathrm{SD})$ and compared between groups using a $t$-test. Non-normally distributed measurement data were expressed as the median (25th percentile, 75th percentile, and M ([Q25, Q75]) and compared between groups using a non-parametric test. The difference in postoperative AF-free survival time was analyzed with a Kaplan-Meier survival analysis using the Breslow (generalized Wilcoxon) test. All the data analyses were conducted using SPSS 25.0 statistical software (International Business Machines Corporation, Chicago, America). A value of $p<0.05$ (twoway) was considered statistically significant.

\section{Results}

\section{Baseline Characteristics of the Three Groups of Patients}

A total of 307 patients who met the inclusion criteria were randomly divided into three groups and were followed up. During the period of 12 months, 14 patients were lost to follow-up or withdrew; 293 patients finally completed follow-up. There were 92 patients in Group 1: 60.9\% of them were males, their mean age was 62 (54-68) years old, $84.8 \%$ of them had paroxysmal AF. There were 99 patients in Group 2: $51.5 \%$ of them were males, their mean age was 62 (54-66) years old, $88.9 \%$ of them had paroxysmal AF. There were 102 patients in Group 3: 59.8\% of them were males, their mean age was 60 (52-66) years old, $91.2 \%$ of them had paroxysmal AF. The differences in baseline data between the three groups were not statistically significant (Table 1).

\section{Comparison of Operation Conditions Among the Three Groups}

The mean operation time was $130(125-140)$ minutes in Group 1, 140 (130-150) minutes in Group 2, and 150 (140-166) minutes in Group 3 ( $\mathrm{P}<0.001)$. The comparison between Group 1 and Group 2 revealed $\mathrm{P}=$ 0.006, while the comparisons between Group 1 and 


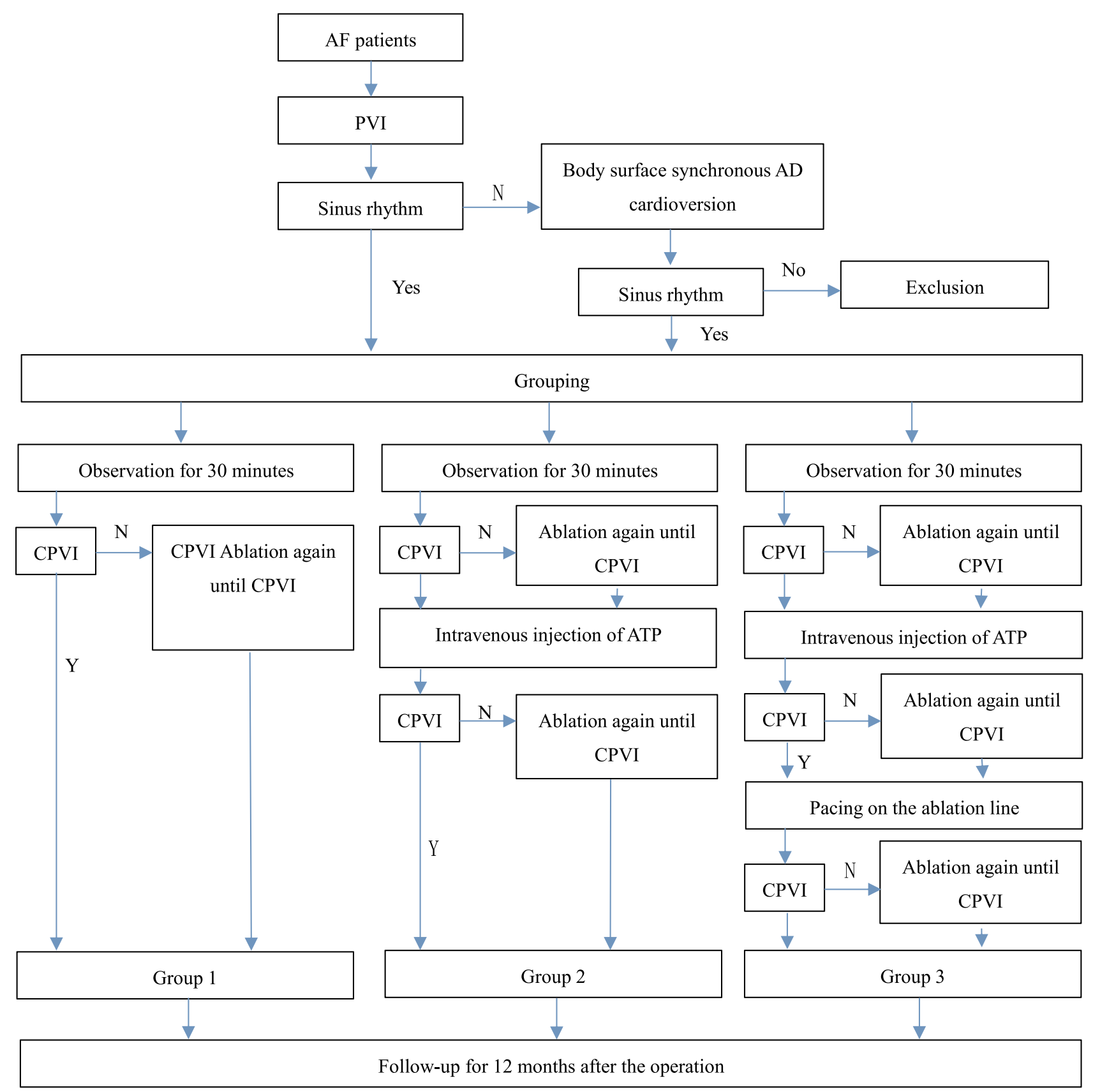

Figure I Study grouping flow chart.

Group 3, and Group 2 and Group 3 revealed $\mathrm{P}<0.001$. The mean X-ray exposure time of the three groups were 7.9 (5.2-12.2) minutes, $7.6(4.5-10.8)$ minutes, and 7.6 (5.0-10.9) minutes, respectively, $\mathrm{P}=0.522$. The X-ray exposure doses of the three groups were 163.1 (100.7-252.0) mGy, 135.9 (96.6-196.1) mGy, and 170.7 (97.0-247.1) $\mathrm{mGy}$, respectively, $\mathrm{P}=0.236$ (Table 2). In Group 1, one patient had acute cardiac tamponade and bleeding was stopped after pericardiocentesis and drainage by catheterization. Three patients in Group 2 and two patients in Group 3 had different degrees of ecchymosis at the inguinal blood-vessel puncture site. Pseudoaneurysms and arteriovenous fistulas were excluded by local B-ultrasonography, and the ecchymosis gradually disappeared after application of a local compression bandage. The difference between the three groups was not statistically significant: $\mathrm{P}=0.783$. During the operation and follow-up period, no surgery- 
Table I Basic Data of Patients

\begin{tabular}{|c|c|c|c|c|}
\hline & No. I Group $(n=92)$ & No. 2 Group $(n=99)$ & No. 3 Group $(n=102)$ & $P$ value \\
\hline Male (cases(\%)) & $56(60.9 \%)$ & $5 \mathrm{I}(5 \mathrm{I} .5 \%)$ & $6 \mathrm{I}(59.8 \%)$ & 0.351 \\
\hline Age (years) & $62(54-68)$ & $62(54-66)$ & $60(52-66)$ & 0.478 \\
\hline \multicolumn{5}{|l|}{ AF type } \\
\hline Paroxysmal AF (cases [\%]) & $78(84.8 \%)$ & $88(88.9 \%)$ & $93(91.2 \%)$ & 0.375 \\
\hline Persistent AF (cases [\%]) & $14(\mid 5.2 \%)$ & $\mathrm{II}(\mathrm{II} . \mathrm{I} \%)$ & $9(8.8 \%)$ & \\
\hline Course of $\mathrm{AF}$ & $24(|2-6|)$ & $13(5-48)$ & $18(4-60)$ & 0.282 \\
\hline Hypertension (cases) & $43(46.7 \%)$ & $5 \mathrm{I}(5 \mathrm{I} .5 \%)$ & $4 \mathrm{I}(40.2 \%)$ & 0.271 \\
\hline Left ventricular hypertrophy (cases) & $29(31.5 \%)$ & $31(31.3 \%)$ & $33(32.3 \%)$ & 0.986 \\
\hline Coronary heart disease (cases) & $8(8.7 \%)$ & $4(4.0 \%)$ & $4(3.9 \%)$ & 0.287 \\
\hline Diabetes (cases) & $\mathrm{II}(\mathrm{I} 2.0 \%)$ & $15(15.2 \%)$ & $16(15.7 \%)$ & 0.730 \\
\hline \multicolumn{5}{|l|}{ NYHA } \\
\hline I level (cases) & $5 \mathrm{I}(55.4 \%)$ & $64(64.6 \%)$ & $63(61.8 \%)$ & $0.4 \mid 4$ \\
\hline II level (cases) & $4 \mathrm{I}(44.6 \%)$ & $35(35.4 \%)$ & $39(38.2 \%)$ & \\
\hline BNP & $112(47-300)$ & $110(45-265)$ & $\mid 28(5 \mid-248)$ & 0.820 \\
\hline BMI $\left(\mathrm{kg} / \mathrm{m}^{2}\right)$ & $23.8 \pm 2.8$ & $24.3 \pm 3.2$ & $24.1 \pm 3.2$ & 0.539 \\
\hline $\mathrm{CrCl}(\mathrm{mL} / \mathrm{min})$ & $83.1(69.3-101.5)$ & $85.7(71.4-105.1)$ & $85.5(69.1-97.5)$ & 0.725 \\
\hline \multicolumn{5}{|l|}{$\mathrm{CHA}_{2} \mathrm{DS}_{2}$-VASc score (cases) } \\
\hline $0 \sim 1$ points & $39(42.4 \%)$ & $44(44.4 \%)$ & $48(47.1 \%)$ & 0.806 \\
\hline$\geq 2$ points & $53(57.6 \%)$ & $55(55.6 \%)$ & $54(52.9 \%)$ & \\
\hline \multicolumn{5}{|l|}{ HAS-BLED score (cases) } \\
\hline $0 \sim 2$ points & $85(92.4 \%)$ & $89(89.9 \%)$ & $96(94.1 \%)$ & 0.536 \\
\hline$\geq 3$ points & $7(7.6 \%)$ & $10(10.1 \%)$ & $6(5.9 \%)$ & \\
\hline Smoking history (cases) & $24(26.1 \%)$ & $22(22.2 \%)$ & $26(25.5 \%)$ & 0.796 \\
\hline Previous TIA/ischemic stroke (cases) & $15(16.3 \%)$ & $14(\mid 4.1 \%)$ & $8(7.8 \%)$ & 0.178 \\
\hline Postoperative use of ACE-I or ARB (cases) & $29(31.5 \%)$ & $25(25.3 \%)$ & $23(22.5 \%)$ & 0.131 \\
\hline Postoperative use of statins (cases) & $27(29.3 \%)$ & $22(22.2 \%)$ & $22(21.6 \%)$ & 0.382 \\
\hline Postoperative use of $\beta$-receptor blockers (cases) & $26(28.3 \%)$ & $17(17.2 \%)$ & $20(19.6 \%)$ & 0.149 \\
\hline Postoperative use of spironolactone (cases) & $5(5.4 \%)$ & $2(2.0 \%)$ & $2(2.0 \%)$ & 0.401 \\
\hline
\end{tabular}

Table 2 Comparison of Operation Time and Others Among 3 Groups

\begin{tabular}{|l|c|c|c|c|}
\hline & No.I Group(n=92) & No. 2 Group(n=99) & No. 3 Group(n=102) & P value \\
\hline Operation time (min) & $130(125-140)$ & $140(130-150)$ & $150(140-166)$ & 0.000 \\
\hline X-ray exposure time (min) & $7.9(5.2-12.2)$ & $7.6(4.5-10.8)$ & $7.6(5.0-10.9)$ & 0.522 \\
\hline X-ray exposure dose (mGy) & $163.1(100.7-252.0)$ & $135.9(96.6-196.1)$ & $170.7(97.0-247.1)$ & 0.236 \\
\hline
\end{tabular}

related complications - such as left-atrial-esophageal fistula, phrenic-nerve injury, symptomatic stroke, or hemorrhagic stroke-occurred.
The 92 patients in Group 1 were observed for 30 minutes after the initial PVI. In $38 / 92$ patients $(41.3 \%)$, electrical conduction between $118 / 384$ patients $(30.7 \%)$ of 
PV-LA was seen to recover and supplemental ablation was used until PVI was repeated.

The 99 patients in Group 2 were observed for 30 minutes after the initial PVI. In 45/99 patients (45.5\%) (observed [+]), electrical conduction between 153/425 (36.0\%) of PV-LA was seen to recover and supplemental ablation was used until PVI was performed again. Then the patients were intravenously injected with ATP: in 40/ 99 patients $(40.4 \%)$ (observed $[+]$ ), electrical conduction between 121/425 (28.5\%) of PV-LA was seen to recover. Among these 40 patients, in the observed (+) cohort, in 12/ 40 patients (30.0\%), electrical conduction between 39/192 (16.7\%) of PV-LA was detected; in the observed (-) cohort, in 28/40 patients (70.0\%), electrical conduction between 89/233 (38.2\%) of PV-LA was seen to recover. After completing the above two steps, in $73 / 99$ patients (73.7\%), electrical conduction between 242/425 (56.9\%) of PV-LA was detected and supplemental ablation was performed until PVI was repeated.

The 102 patients in Group 3 were observed for 30 minutes after the initial PVI. In 44/102 patients (43.1\%) (observed [+]), electrical conduction between 136/431 (31.6\%) of PV-LA was seen to recover and supplemental ablation was performed until PVI was repeated. Then the patients were intravenously injected with ATP and in 43/ 102 patients $(42.2 \%)$ (ATP $[+])$, electrical conduction between 134/431 (31.1\%) of PV-LA was detected to recover. Among the 43 patients in the observed $(+)$ cohort, in $16 / 43$ patients $(37.2 \%)$ electrical conduction between 48/186 (25.8\%) of PV-LA was detected; in the observed (-) cohort, in 27/43 patients (62.8\%) electrical conduction between $86 / 245$ (35.1\%) of PV-LA was seen to recover. Finally, pacing was performed on the ablation line and in $35 / 102$ patients $(34.3 \%)$ (pacing $[+]$ ), electrical conduction between 109/431 (25.3\%) of PV-LA was detected. Among these 51 patients, in the observed (+) and ATP $(+)$ cohort, in $4 / 35$ patients (11.4\%) electrical conduction between 10 / $68(16.7 \%)$ of PV-LA was detected to recover; in the observed (+) and ATP (-) cohort, in 9/35 patients (25.7\%) electrical conduction between 28/118 (23.7\%) of PV-LA was detected; in the observed $(-)$ and ATP $(+)$ cohort, in 10/35 patients (28.6\%) electrical conduction between 33/114 (28.9\%) of PV-LA was seen to recover; in the observed (-) and $\operatorname{ATP}(-)$ cohort, in 12/35 patients (34.3\%) electrical conduction between 38/114 (33.3\%) of PV-LA was detected. After completing the above three steps, in $83 / 102$ patients $(81.4 \%)$, electrical conduction between $260 / 431(60.3 \%)$ of PV-LA was detected to recover, and supplemental ablation was performed until PVI was repeated.

\section{Comparison of Echocardiography Indices Among the Three Groups Before the Operation}

The left atrial sizes of the three groups were $37.4 \pm$ $6.2 \mathrm{~mm}, 36.9 \pm 5.5 \mathrm{~mm}$, and $37.3 \pm 5.8 \mathrm{~mm}$, respectively, $\mathrm{P}=0.787$. The pulmonary arterial pressure was 27.0 (18.0-35.0) mmHg, 27.0 (18.0-33.0) $\mathrm{mmHg}$, and 25.0 (18.0-31.3) $\mathrm{mmHg}$, respectively, $\mathrm{P}=0.610$. The left-ventricular ejection fraction was $66.0 \%(62.0-71.8 \%), 67.5 \%$ (62.0-$70.7 \%$ ), and $66.6 \%$ (62.0-71.1\%), respectively, $\mathrm{P}=0.949$. The thickness of the interventricular septum was 10.4 (9.5-11.7) $\mathrm{mm}, \quad 10.3 \quad(9.6-11.3) \quad \mathrm{mm}, \quad$ and 10.0 (9.2-11.7) $\mathrm{mm}$, respectively, $\mathrm{P}=0.635$. The leftventricular posterior wall thickness was 10.0 (9.1-11.1) mm, $9.8 \quad(9.1-10.6) \quad \mathrm{mm}, \quad$ and 9.6 (8.8-10.4) mm, respectively, $\mathrm{P}=0.113$ (Table 3).

\section{Comparison of Echocardiography Indices of the Three Groups at 12 Months After the Operation}

The left atrial sizes of the three groups were $34.9 \pm$ $7.1 \mathrm{~mm}, 33.5 \pm 6.6 \mathrm{~mm}$, and $33.0 \pm 6.1 \mathrm{~mm}$, respectively, $\mathrm{P}=0.136$. The pulmonary arterial pressure was 28.0 (20.0-34.7) mmHg, 25.0 (18.0-31.0) mmHg, and 20.0 (18.0-27.0) $\mathrm{mmHg}$, respectively, $\mathrm{P}<0.001$. A comparison between Groups 1 and 2 revealed $\mathrm{P}=0.227$, a comparison between Groups 1 and 3 revealed $\mathrm{P}<$ 0.001 , and a comparison between Groups 2 and 3 revealed $\mathrm{P}=0.040$. The left-ventricular ejection fraction was $66.8 \%$ (62.5-70.2\%), 67.0\% (63.8-70.2\%), and 67.6\% (64.5-$70.7 \%$ ), respectively, $\mathrm{P}=0.185$. The thickness of the interventricular septum was $10.1(9.2-11.6) \mathrm{mm}, 9.9$ (9.1-10.8) $\mathrm{mm}$, and $9.6(8.9-10.9) \mathrm{mm}$, respectively, $\mathrm{P}=$ 0.167 . The left-ventricular posterior wall thickness was 9.6 (8.9-10.7) $\mathrm{mm}, \quad 9.3 \quad(8.9-10.2) \mathrm{mm}, \quad$ and 9.2 (8.6-10.3) mm, respectively, $\mathrm{P}=0.119$ (Table 3).

\section{Comparison of Echocardiography Indices Among the Three Groups Before the Operation and at 12 Months After the Operation}

In Group 1, after the operation the left-atrial thickness $(P<0.001)$ and left-ventricular posterior wall thickness 
Table 3 Comparison of Echocardiography Indexes Among 3 Groups

\begin{tabular}{|c|c|c|c|c|c|}
\hline & & $\begin{array}{l}\text { No. I Group } \\
\quad(n=92)\end{array}$ & $\begin{array}{l}\text { No. } 2 \text { Group } \\
(n=99)\end{array}$ & $\begin{array}{c}\text { No. } 3 \text { Group } \\
(n=102)\end{array}$ & $P$ value \\
\hline \multirow[t]{5}{*}{ Before operation } & Left atrium $(\mathrm{mm})$ & $37.4 \pm 6.2$ & $36.9 \pm 5.5$ & $37.3 \pm 5.8$ & 0.787 \\
\hline & Pulmonary arterial pressure $(\mathrm{mmHg})$ & $27.0(18.0-35.0)$ & $27.0(18.0-33.0)$ & $25.0(|8.0-3| .3)$ & 0.610 \\
\hline & Left ventricular ejection fraction (\%) & $66.0(62.0-71.8)$ & $67.5(62.0-70.7)$ & $66.6(62.0-71.1)$ & 0.949 \\
\hline & Interventricular septum thickness (mm) & $10.4(9.5-11.7)$ & $10.3(9.6-11.3)$ & $10.0(9.2-11.7)$ & 0.635 \\
\hline & Left ventricular posterior wall thickness $(\mathrm{mm})$ & $10.0(9.1-11.1)$ & $9.8(9.1-10.6)$ & $9.6(8.8-10.4)$ & 0.113 \\
\hline \multirow[t]{5}{*}{ Post operation } & Left atrium $(\mathrm{mm})$ & $34.9 \pm 7.1$ & $33.5 \pm 6.6$ & $33.0 \pm 6.1$ & 0.136 \\
\hline & Pulmonary arterial pressure $(\mathrm{mmHg})$ & $28.0(20.0-34.7)$ & $25.0(\mid 8.0-31.0)$ & $20.0(18.0-27.0)$ & 0.000 \\
\hline & Left ventricular ejection fraction (\%) & $66.8(62.5-70.2)$ & $67.0(63.8-70.2)$ & $67.6(64.5-70.7)$ & 0.185 \\
\hline & Interventricular septum thickness (mm) & $10.1(9.2-11.6)$ & $9.9(9.1-10.8)$ & $9.6(8.9-10.9)$ & 0.167 \\
\hline & Left ventricular posterior wall thickness $(\mathrm{mm})$ & $9.6(8.9-10.7)$ & $9.3(8.9-10.2)$ & $9.2(8.6-10.3)$ & 0.119 \\
\hline
\end{tabular}

$(\mathrm{P}=0.013)$ were decreased and there was no significant difference in pulmonary artery pressure $(\mathrm{P}=$ 0.296), left-ventricular ejection fraction $(P=0.629)$, or the thickness of the interventricular septum $(\mathrm{P}=$ 0.053). In Group 2, after the operation the left-atrial thickness $(\mathrm{P}<0.001)$, left-ventricular posterior wall thickness $(\mathrm{P}=0.028)$, and the thickness of the interventricular septum $(P=0.006)$ were decreased, while there was no significant difference in pulmonary artery pressure $(\mathrm{P}=0.284)$ and left-ventricular ejection fraction $(P=0.626)$. In Group 3 , there were significant differences in left-atrial size, pulmonary artery pressure, and the thickness of the interventricular septum ( $\mathrm{P}<0.001$ for all), while there were significant differences in the left-ventricular ejection fraction and leftventricular posterior wall thickness $(P=0.007$ and 0.004 , respectively) (Table 3 ).

\section{Analysis of the Incidence of AF and AF- Free Survival in the Three Groups}

At the end of the follow-up, 12 months after the operation, 28 patients in Group 1 (30.4\%), 19 patients in Group 2 (19.2\%), and 10 patients in Group 3 (9.8\%) showed recurrence of AF. The differences between the three groups were statistically significant $(\mathrm{P}=0.001)$ : the difference between Groups 1 and 2 was not statistically significant $(\mathrm{P}=0.070)$, the difference between Groups 1 and 3 was statistically significant $(\mathrm{P}<$ 0.001 ) (three pairwise comparisons were required, and the corrected inspection level $\alpha=0.05 / 3=0.0167$ ), and the difference between Groups 2 and 3 was not statistically significant $(\mathrm{P}=0.058)$ (see Table 4). Of these recurrent patients, eight patients $(29.6 \%)$ in Group 1, 10 patients $(45.5 \%)$ in Group 2, and seven patients (46.7\%) in Group 3 underwent secondary radiofrequency ablation (RFA). The

Table 4 Comparison of the Number of Cases of Recurrent AF Among 3 Groups

\begin{tabular}{|c|c|c|c|c|c|c|}
\hline & Recurrence & No Recurrence & Total & $\begin{array}{c}\text { Recurrence } \\
\text { Rate }\end{array}$ & $\chi^{2}$ & $p$ \\
\hline Group I & 28 & 64 & 92 & $30.4 \%$ & 3.249 & $0.07 I$ \\
\hline Group 2 & 19 & 80 & 99 & $19.2 \%$ & & \\
\hline Group I & 28 & 64 & 92 & $30.4 \%$ & $|3.07|$ & $0.000 *$ \\
\hline Group 3 & 10 & 92 & 102 & $9.8 \%$ & & \\
\hline Group 2 & 19 & 80 & 99 & $19.2 \%$ & 3.586 & 0.058 \\
\hline Group 3 & 10 & 92 & 102 & $9.8 \%$ & & \\
\hline
\end{tabular}

Note: *In this case, pairings should be performed three times, and the calibration test level is $=0.05 / 3=0.0167$. 
difference between the three groups was not statistically significant $(\mathrm{P}=0.441)$.

In terms of postoperative $\mathrm{AF}$ free survival time of patients, the comparison between Groups 1 and 2 revealed $\chi^{2}=4.582$ and $\mathrm{P}=0.032$, the comparison between Groups 1 and 3 revealed $\chi^{2}=16.787$ and $\mathrm{P}<0.001$, and the comparison between Groups 2 and 3 revealed $\chi^{2}=4.948$ and $\mathrm{P}=0.026$. In this study, the possible factors influencing AF recurrence after the operation-such as AF type, course of AF, gender, age, body mass index, creatinineclearance rate, preoperative left-atrial size, pulmonary artery pressure, ejection fraction, left-ventricular hypertrophy, hypertension, diabetes mellitus, and coronary heart disease - were used for Cox's proportional hazard regression model analysis. A stepwise regression model test revealed that the difference was statistically significant, $\chi^{2}=32.508, \mathrm{P}=0.019$. Study grouping was a risk factor for recurrence of $\mathrm{AF}, \mathrm{P}=0.002$ : compared with Group 3, the hazard ratio (HR) of AF recurrence in Group 2 was $2.208,95 \%$ confidence interval $(\mathrm{CI})(1.008-4.338), \mathrm{P}=$ 0.048 , and the HR of AF recurrence in Group 1 was 3.717, 95\% CI (1.760-7.850), $\mathrm{P}=0.001$. The course of AF was also a risk factor for recurrence of $\mathrm{AF}$ : the longer the course of AF, the higher the risk of $\mathrm{AF}$ recurrence, $\mathrm{HR}=$ $1.006,95 \%$ CI (1.002-1.010), $\mathrm{P}=0.005$.

\section{Discussion}

Radiofrequency catheter ablation is an effective treatment for $\mathrm{AF}$, and its cornerstone is persistent and effective PVI. ${ }^{3}$ The recurrence rate of AF after surgery varies, and the recovery of PV-LA electrical conduction is the main cause of the recurrence of AF after ablation. Ensuring the permanence and long-term persistence of PVI is the key to reducing the recurrence of $\mathrm{AF}$.

The process of catheter ablation may, in some patients, cause only reversible or non-transmural damage to atrial myocytes. After PVI is achieved, if the observation time is prolonged, the recovery of PV-LA electrical conduction can be observed. Some scholars have shown that the recovery of PV-LA electrical conduction was observed at 60 minutes or more after PVI. ${ }^{4}$ Cheema et al revealed in their study ${ }^{5}$ that, when an observation was made for 60 minutes after PVI, the PV-LA electrical conduction was recovered in more than $30 \%$ of the patients. Ablation was repeated to achieve PVI, and the success rate of the surgery was significantly higher than that of patients without observation time after the initial PVI. Although prolonged observation time after PVI can detect more patients with recovery of PV-LA electrical conduction, it has not been determined how long the observation time should be, and it is obviously unrealistic to require a long observation time after surgery. At present, the observation time commonly used in electrophysiological centers is 30 minutes. In the present study, patients in group 1 were observed for 30 minutes after the initial PVI. In 28/92 patients (30.4\%), PV-LA electrical conduction between 101 and 378 (26.7\%) was seen to recover, and ablation was repeated until PVI recurred. The success rate of surgery was $69.6 \%$ at 12 months after the operation. This is similar to that reported in the literature. ${ }^{6}$

\section{The Energy of RFA Damages Cells}

The resting potential of injured cells is raised to a level higher than the excitability threshold, rendering the cells unable to depolarize and conduct. The outward potassium current activated during the recovery phase of the action potential of cardiomyocytes is beneficial to the hyperpolarization of the cell membrane, maintaining the excitability of the cells. This outward potassium current is also damaged by the ablation energy. However, adenosine can enhance this outward potassium current, promoting the hyperpolarization of the cell membrane potential and maintaining excitability. It can also activate sodium channels and increase the maximum depolarization rate in phase 0 of the cellular action potential. However, adenosine cannot restore the excitability of cells that have been completely or irreversibly damaged. Based on this, after radiofrequency catheter ablation, adenosine can be used to locate reversible injuries, allowing re-ablation of these sites to reduce the recurrence of $\mathrm{AF}^{7,8}$

In the $\mathrm{ADVICE}^{9,10}$ study, 534 patients with paroxysmal AF undergoing RFA were enrolled and observed for 20 minutes after PVI then injected with adenosine. The patients were divided into three groups - recovery + ablation, recovery + no ablation, and no recovery - according to whether or not there was recovery of PV-LA electrical conduction after the adenosine injection. The patients were followed up for 12 months after the operation, and the success rate of the three groups was $69.4 \%, 42.3 \%$, and $55.7 \%$, respectively.

In their study, Yamane et al ${ }^{11}$ enrolled 75 patients with paroxysmal AF. Patients were injected with adenosine and observed for 30 minutes after the initial PVI, and then ablation was performed at all sites where it was found that the electrical conduction between PV and LA had 
recovered. This operation was repeated three times until 90 minutes after the initial PVI. The average follow-up time after surgery was 370 days, and the success rate of the operation was $92 \%$.

The first innovation of the current study is that 88 patients (88.9\%) with paroxysmal $\mathrm{AF}$ and 11 patients (11.1\%) with persistent AF were included in group 2. Patients with persistent AF who could not maintain sinus rhythm after the initial PVI + cardioversion were excluded. Thus, the ablation strategy for patients with persistent AF and paroxysmal AF was the same. Patients were injected with adenosine and observed for 30 minutes after the initial PVI. In 38/99 patients (38.4\%), PV-LA electrical conduction between 125 and 407 (30.7\%) was seen to recover, and ablation was repeated until PVI recurred. The success rate of the surgery was $80.8 \%$ at 12 months after the operation.

However, although prolonged observation time alone after PVI can detect the recovery of electrical conduction between more PVs and LA, it has not been determined how long the observation time should be. Most scholars believe that it is unrealistic to require a 90-minute observation time for daily work. ${ }^{3}$ In the present study, the observation time after the initial PVI was 30 minutes, far less than that proposed by Yamane et al (90 minutes), which may be the reason for the success rate of the present study being lower than that of Yamane et al.

In the present study, a radiofrequency catheter with pressure sensing and cold saline perfusion obtained from Johnson \& Johnson Company (NJ, USA) was used. Realtime pressure monitoring can ensure close contact between the catheter and the tissue during ablation, increasing the ratio of irreversible cell damage caused by the first ablation. This may be the reason that, in this study, the recovery rate of PV-LA electrical conduction after the initial PVI was lower while the postoperative success rate was higher than those seen in the ADVICE study.

However, while the cause of the recovery of PV-LA electrical conduction after PVI was related to the occult conduction revealed by the ATP injection, it was also related to the ablation-end transmural injury of the pulmonary vein vestibule and heterogeneity caused by the pulmonary vein muscle sleeve's extending to the pulmonary vein. ${ }^{12}$ Therefore, other techniques, such as determining viable myocardium or potential gaps by pacing along the PVI line at the distal end of the ablation catheter, need to be combined ${ }^{13}$ to reduce the recurrence of AF after PVI.
Detecting excitable gaps by strong electrical stimulation on the ablation line is helpful to achieve the permanent electrical isolation of pulmonary veins. A two-center prospective randomized controlled study conducted by Steven et $\mathrm{al}^{2}$ revealed that, compared with only aiming to achieve a two-way block of the pulmonary vein, the strategy of using pacing on the ablation line (current: 10 $\mathrm{mA}$; pulse width: $2 \mathrm{~ms}$ ) without capture as an additional goal can significantly improve the short-term effects of a single operation $(52.0 \%$ vs. $82.7 \%)$. Kogawa et al revealed in their study ${ }^{14}$ that the distribution of latent conduction sites between PV and LA detected by an injection of adenosine and pacing capture was not consistent. This suggests that these two methods may have different mechanisms for the recovery of latent conduction between PV and LA. Pedrote et $\mathrm{al}^{15}$ suggested that adenosine provocation test + pacing capture + supplemental ablation may help reduce the recurrence of AF.

The second innovation of this study is that patients in group 3 were injected with adenosine + pacing capture + observation for 30 minutes after the initial PVI. In 46/102 patients (45.1\%), PV-LA electrical conduction between 142 and 417 (34.1\%) was seen to recover, and ablation was repeated until PVI recurred. The success rate of the surgery was $90.2 \%$ at 12 months after the operation. This suggests that, when patients with paroxysmal AF and persistent AF receive adenosine injection + pacing capture + observation for 30 minutes after the initial PVI and then undergo ablation again at all sites where PV-LA electrical conduction is seen to recover, the recurrence rate of $\mathrm{AF}$ after the operation can be significantly reduced.

The third innovation of this study is that the three methods were compared directly. At the end of the followup period, 12 months after the operation, 28 patients in group 1 (30.4\%), 19 patients in group $2(19.2 \%)$, and 10 patients in group 3 (9.8\%) showed a recurrence of AF. The difference among the three groups was statistically significant $(p=0.001)$, the differences between groups 1 and 2 and between groups 2 and 3 were not statistically significant, and the difference between groups 1 and 3 was statistically significant $(\mathrm{p}<0.001)$. The Kaplan-Meier survival function without atrial tachyarrhythmia $(\mathrm{AF}$, atrial flutter, or atrial tachycardia) was investigated with a Breslow (generalized Wilcoxon) test. A pairwise comparison was conducted between the three groups, and the difference was statistically significant. This suggests that, compared with the treatment of 30 minutes observation + supplemental ablation, the treatment of 30 minutes 
observation + injection of adenosine + pacing capture + supplemental ablation can reduce the recurrence of AF and prolong the atrial arrhythmia-free survival time. Although the operation time of the latter was significantly prolonged, there was no difference in X-ray exposure time and exposure dose, and no increase in perioperative complications.

The fourth innovation of this study is that the possible factors influencing AF recurrence after operation, such as AF type, course of AF, gender, age, body mass index, creatinine clearance rate, preoperative left atrial size, pulmonary artery pressure, ejection fraction, left ventricular hypertrophy, hypertension, diabetes mellitus and coronary heart disease, were used for Cox's proportional hazard regression model analysis. It was found that two factors were affecting the recurrence of AF. The first was the surgical method: compared with group 3, HR of AF recurrence in group 2 was 2.208 (95\% CI: 1.008-4.338, $\mathrm{p}=0.048)$, and $\mathrm{HR}$ of $\mathrm{AF}$ recurrence in group 1 was 3.717 (95\% CI: $1.760-7.850, \mathrm{p}=$ $0.001)$. The second factor was the course of AF: the longer the course of AF, the higher the risk of AF recurrence $(\mathrm{HR}=$ $1.006,95 \% \mathrm{CI}: 1.002-1.010, \mathrm{p}=0.005)$. AF is a progressive disease, often progressing from paroxysmal AF to persistent AF. AF can cause atrial remodeling, including electrical remodeling and structural remodeling. The latter makes AF more likely to occur and persist. The longer the course of AF, the more obvious the atrial remodeling, and more changes in the atrial matrix may be involved in the occurrence and persistence of AF. Therefore, the course of AF is an important factor affecting the recurrence of AF after surgery. This suggests that RFA should be performed as early as possible after the diagnosis of AF since, by doing so, the recurrence rate of AF after surgery can be significantly reduced.

The differences in left atrial size, mean pulmonary artery pressure, left ventricular ejection fraction, thickness of the left ventricular posterior wall, and thickness of the interventricular septum among the three groups were not statistically significant. One year after the operation, the echocardiography was re-examined, and the results revealed that there was no significant difference among the three groups except in mean pulmonary artery pressure. It is speculated that the decrease in mean pulmonary artery pressure after the operation may be related to the sequential contraction of the atrium and ventricle, the recovery of left atrial systolic function, and the decrease in left atrial end-systolic pressure in sinus rhythm compared with AF. To gain a clearer understanding of the mechanism, further research is required.
In summary, for patients with paroxysmal AF, and some enrolled patients with persistent AF, after PVI, the endpoint assessment method of 30 minutes observation + adenosine injection + pacing capture + supplemental ablation can significantly reduce the recurrence rate of AF 12 months after the operation and improve the success rate of RFA of AF. Although the operation time was significantly prolonged, there was no difference in X-ray exposure time and exposure dose, and no increase in perioperative complications. Moreover, there was no change in heart function, such as left atrial size and left ventricular ejection fraction. Therefore, as the cornerstone of RFA of AF is permanent and irreversible PVI, the endpoint assessment method of 30 minutes observation + adenosine injection + pacing capture + supplemental ablation is an effective and safe way to achieve this goal.

This study has the following limitations, however. It was a single-center study that conducted retrospective data analysis, and therefore there may be a certain degree of bias. Multi-center clinical studies with large samples are needed to obtain data that are more in line with the actual clinical situation. Furthermore, some patients had transient asystole after the adenosine injection, and some positions of PV-LA electrical conduction recovery may have been missed. For patients with recurrent AF who underwent reablation, there is no accurate comparison of the position of PV-LA electrical conduction recovery. Therefore, it is necessary to determine whether all the methods (30 minutes observation, adenosine injection, and pacing capture) can detect the position of PV-LA electrical conduction recovery, and which order is most beneficial.

\section{Acknowledgments}

We would like to acknowledge the hard and dedicated work of all the staff that implemented the intervention and evaluation components of the study.

\section{Funding}

This work was supported by the Fujian Provincial Natural Science Foundation (Grant No. 2017Y0039), Fujian Province Medical Innovation Project (Grant No. 2012CXB-19), Fujian Provincial Natural Science Foundation (Grant No. 2018J01298) and Young and Middle-Aged Scholars Foundation of Fujian Province Health Bureau (Grant No. 2017-ZQN-29).

\section{Disclosure}

The authors declare that they have no competing interests. 


\section{References}

1. Huang C, Zhang S, Huang D, Hua W. Current knowledge and management recommendations of atrial fibrillation:2018. Chin J Cardiac Pacing Electrophysiol. 2018;32(4):315-365.

2. Steven D, Sultan A, Reddy V, et al. Benefit of pulmonary vein isolation guided by loss of pace capture on the ablation line: results from a prospective 2-center randomized trial. $J$ Am Coll Cardiol. 2013;62 (1):44-50. doi:10.1016/j.jacc.2013.03.059

3. Dallaglio PD, Betts TR, Ginks M, Bashir Y, Anguera I, Rajappan K. The role of adenosine in pulmonary vein isolation: a critical review. Cardiol Res Pract. 2016;2016:8632509. doi:10.1155/2016/8632509

4. Wang XH, Liu X, Sun YM, et al. Early identification and treatment of PV re-connections: role of observation time and impact on clinical results of atrial fibrillation ablation. Europace. 2007;9(7):481-486. doi:10.1093/europace/eum101

5. Cheema A, Dong J, Dalal D, et al. Incidence and time course of early recovery of pulmonary vein conduction after catheter ablation of atrial fibrillation. $J$ Cardiovasc Electrophysiol. 2007;18(4):387-391. doi:10.1111/j.1540-8167.2007.00760.x

6. Kumagai K, Naito S, Nakamura K, et al. ATP-induced dormant pulmonary veins originating from the carina region after circumferential pulmonary vein isolation of atrial fibrillation. $J$ Cardiovasc Electrophysiol. $\quad 2010 ; 21(5): 494-500 . \quad$ doi:10.1111/j.1540-8167.20 09.01667.x

7. Datino T, Macle L, Qi X-Y, et al. Mechanisms by which adenosine restores conduction in dormant canine pulmonary veins. Circulation. 2010;121(8):963-972. 107

8. Theis C, Konrad T, Mollnau H, et al. Arrhythmia termination versus elimination of dormant pulmonary vein conduction as a procedural end point of catheter ablation for paroxysmal atrial fibrillation: a prospective randomized trial. Circ Arrhythm Electrophysiol. 2015;8 (5):1080-1087. doi:10.1161/CIRCEP.115.002786
9. Macle L, Khairy P, Verma A, et al.; ADVICE Study Investigators. Adenosine following pulmonary vein isolation to target dormant conduction elim- ination (ADVICE): methods and rationale. Can J Cardiol. 2012;28(2):184-190. doi:10.1016/j.cjca.2011.10.008

10. Macle L, Khairy P, Weerasooriya R, et al.; ADVICE trial investigators. Adenosine-guided pulmonary vein isolation for the treatment of paroxysmal atrial fibrillation: an international, multicentre, randomised superiority trial. Lancet. 2015;386(9994):672-679. doi:10.1016/S0140-6736(15)60026-5

11. Yamane T, Matsuo S, Date T, et al. Repeated provocation of timeand ATP-induced early pulmonary vein reconnection after pulmonary vein isolation: eliminating paroxysmal atrial fibrillation in a single procedure. Circ Arrhythm Electrophysiol. 2011;4(5):601-608. doi:10.1161/CIRCEP.110.960138

12. Chen $\mathrm{C}$, Li D, Ho J, et al. Clinical implications of unmasking dormant conduction after circumferential pulmonary vein isolation in atrial fibrillation using adenosine: a systematic review and meta-analysis. Front Physiol. 2018;9:1861-1874. doi:10.3389/ fphys.2018.01861

13. Schaeffer B, Willems S, Sultan A, et al. Loss of pace capture on the ablation line during pulmonary vein isolation versus "dormant conduction": is adenosine expendable? J Cardiovasc Electrophysiol. 2015;26(10):1075-1080. doi:10.1111/jce.12759

14. Kogawa R, Okumura Y, Watanabe I, et al. Difference between dormant conduction sites revealed by adenosine triphosphate provocation and unipolar pace-capture sites along the ablation line after pulmonary vein isolation. Int Heart J. 2016;57(1):25-29. doi:10.1536/ihj.15-231

15. Pedrote A, Acosta J, Jáuregui-Garrido B, Frutos-López M, AranaRueda E. Paroxysmal atrial fibrillation ablation: achieving permanent pulmonary vein isolation by point-by-point radiofrequency lesions. World J Cardiol. 2017;9(3):230-240. doi:10.4330/wjc.v9.i3.230
International Journal of General Medicine

\section{Publish your work in this journal}

The International Journal of General Medicine is an international, peer-reviewed open-access journal that focuses on general and internal medicine, pathogenesis, epidemiology, diagnosis, monitoring and treatment protocols. The journal is characterized by the rapid reporting of reviews, original research and clinical studies across all disease areas. The manuscript management system is completely online and includes a very quick and fair peer-review system, which is all easy to use. Visit http://www.dovepress.com/ testimonials.php to read real quotes from published authors. 